\title{
Differentiation of idiopathic spinal cord herniation from dorsal arachnoid webs on MRI and CT myelography
}

\author{
Randall Schultz Jr., MD, ${ }^{1}$ Andrew Steven, MD, ${ }^{1}$ Aaron Wessell, MD, ${ }^{2}$ Nancy Fischbein, MD, ${ }^{3}$ \\ Charles A. Sansur, MD, MHSc, ${ }^{2}$ Dheeraj Gandhi, MD, ${ }^{1}$ David Ibrahimi, MD, ${ }^{2}$ and \\ Prashant Raghavan, MBBS ${ }^{1}$
}

Departments of ${ }^{1}$ Diagnostic Radiology and Nuclear Medicine, and ${ }^{2}$ Neurosurgery, University of Maryland Medical Center, Baltimore, Maryland; and ${ }^{3}$ Department of Radiology, Stanford University, Stanford, California

\begin{abstract}
OBJECTIVE Dorsal arachnoid webs (DAWs) and spinal cord herniation (SCH) are uncommon abnormalities affecting the thoracic spinal cord that can result in syringomyelia and significant neurological morbidity if left untreated. Differentiating these 2 entities on the basis of clinical presentation and radiological findings remains challenging but is of vital importance in planning a surgical approach. The authors examined the differences between DAWs and idiopathic SCH on MRI and CT myelography to improve diagnostic confidence prior to surgery.
\end{abstract}

METHODS Review of the picture archiving and communication system (PACS) database between 2005 and 2015 identified 6 patients with DAW and 5 with SCH. Clinical data including demographic information, presenting symptoms and neurological signs, and surgical reports were collected from the electronic medical records. Ten of the 11 patients underwent MRI. CT myelography was performed in 3 patients with DAW and in 1 patient with $\mathrm{SCH}$. Imaging studies were analyzed by 2 board-certified neuroradiologists for the following features: 1) location of the deformity; 2) presence or absence of cord signal abnormality or syringomyelia; 3 ) visible arachnoid web; 4) presence of a dural defect; 5) nature of dorsal cord indentation (abrupt "scalpel sign" vs "C"-shaped); 6) focal ventral cord kink; 7) presence of the nuclear trail sign (endplate irregularity, sclerosis, and/or disc-space calcification that could suggest a migratory path of a herniated disc); and 8) visualization of a complete plane of CSF ventral to the deformity.

RESULTS The scalpel sign was positive in all patients with DAW. The dorsal indentation was C-shaped in 5 of 6 patients with $\mathrm{SCH}$. The ventral subarachnoid space was preserved in all patients with DAW and interrupted in cases of $\mathrm{SCH}$. In no patient was a web or a dural defect identified.

CONCLUSIONS DAW and SCH can be reliably distinguished on imaging by scrutinizing the nature of the dorsal indentation and the integrity of the ventral subarachnoid space at the level of the cord deformity.

https://thejns.org/doi/abs/10.3171/2016.11.SPINE16696

KEY WORDS arachnoid web; cord herniation; MRI; CT myelography; congenital

$\mathrm{D}$ ORSAL arachnoid webs (DAWs) and idiopathic spinal cord herniation $(\mathrm{SCH})$ are uncommon abnormalities affecting the thoracic spinal cord that can result in syringomyelia and significant neurological morbidity if left untreated. ${ }^{11,12}$ DAWs are transverse bands of thickened arachnoid membrane that compress the dorsal aspect of the cord. ${ }^{11,12}$ SCHs are focal ventral or, less commonly, lateral protrusions of a portion, usually a short segment, of the spinal cord through a dural defect ${ }^{7}$ (Fig. 1). Differentiating these 2 entities on the basis of clinical presentation and radiological findings remains challeng- ing but is of vital importance from a surgical perspective. While the surgical access for these lesions is similar, definitive treatment of $\mathrm{SCH}$ requires division of the dentate ligaments such that the spinal cord can be rotated for inspection of the ventral cord and dura, while DAWs are treated with lysis of the web without the need for such exposure or repair.

There are no specific clinical features distinguishing DAWs from SCH. Both entities present with similar symptomatology, ranging from subtle sensory and motor deficits to paraparesis and manifestations of Brown-Séquard

ABBREVIATIONS DAW = dorsal arachnoid web; $\mathrm{SCH}$ = spinal cord herniation; SPACE = sampling perfection with application-optimized contrasts using different flip-angle evolution.

SUBMITTED June 28, 2016. ACCEPTED November 2, 2016.

INCLUDE WHEN CITING Published online March 24, 2017; DOI: 10.3171/2016.11.SPINE16696. 

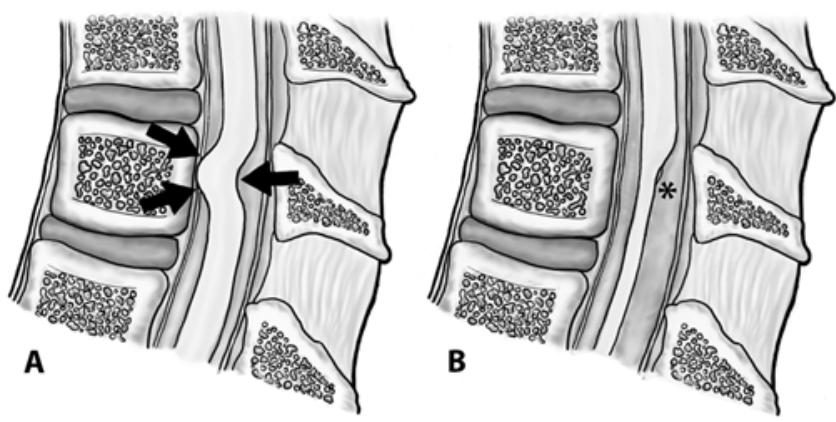

FIG. 1. Illustrations depicting SCH (A) and DAW (B). A: Note the protrusion of the ventral spinal cord through a dural defect, resulting in interruption of the ventral subarachnoid space (arrows) and the C-shaped deformity on the dorsal cord surface. B: A DAW is depicted with a scalpel-sign deformity of the dorsal cord surface (asterisk). Note the preserved ventral subarachnoid space. Copyright Aaron Wessell. Published with permission.

syndrome. The radiological differentiation of these entities may be similar as well. In a review of 27 patients with $\mathrm{SCH}$ by Tekkök, ${ }^{15}$ a correct preoperative diagnosis was made in only 9, with SCH being confused most often with dorsal arachnoid cysts or masses. On MRI and myelography, both entities present as focal anterior displacements of the midthoracic cord with widening of the posterior subarachnoid space. The arachnoid web and the dural defect through which the cord herniates are difficult to directly visualize on imaging. Indeed, some cases of SCH were mistakenly attributed to ventral displacement of the cord by dorsally located arachnoid "cysts," a conclusion stemming from failure to recognize that the dorsal "cyst" was simply an expanded subarachnoid space resulting from the cord being pulled into a dural defect. ${ }^{9}{ }^{13} \mathrm{We}$ therefore sought to examine the differences between these superficially similar entities on MRI and CT myelography in a series of patients who had undergone surgical lysis or release for DAWs or $\mathrm{SCH}$, respectively.

\section{Methods}

This HIPAA-compliant retrospective study was approved by the institutional review board of the University of Maryland Medical Center and the need for informed consent was waived. From a review of the picture archiving and communication system (PACS) database between 2005 and 2015, we identified 6 patients with DAWs and 5 with SCH. Clinical data including demographic information, presenting symptoms and neurological signs, and surgical reports were collected from the electronic medical records. Ten of the 11 patients underwent MRI. CT myelography was performed in 3 patients with DAW and in 1 patient with SCH. MR images were obtained on 1.5-T and 3.0-T MRI machines (Siemens). MRI sequences included standard acquisition fast spin-echo technique with T1- and T2-weighted acquisition. Sagittal T2 sampling perfection with application-optimized contrasts using different flipangle evolution (SPACE) sequences were acquired using the following parameters: TR $1500 \mathrm{msec}$, TE $130 \mathrm{msec}$, flip angle $150^{\circ}$, 1-mm slice thickness, and bandwidth 465 Hz. Axial CT myelographic images were obtained following administration of intrathecal iodinated contrast using
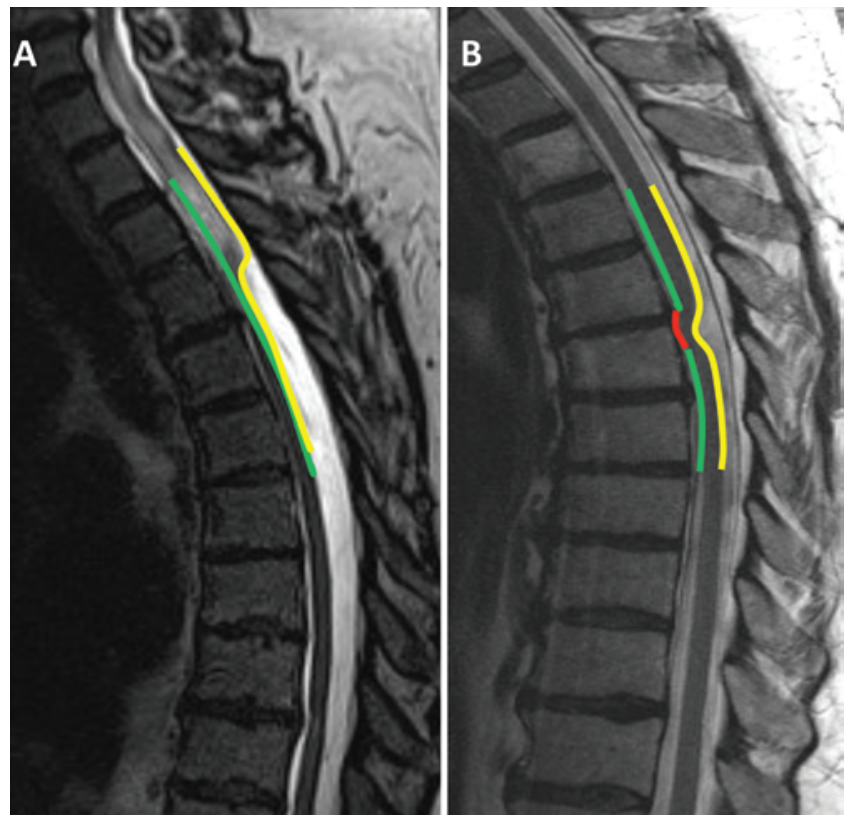

FIG. 2. Sagittal T2-weighted MRI sequences illustrate a thoracic DAW (A) and an SCH (B). The green line follows the course of intact ventral subarachnoid space. The red line depicts the interrupted ventral subarachnoid space due to the cord herniating through a dural defect. The posterior yellow line depicts the shape of the dorsal cord indentation: scalpel-shaped in DAW and $\mathrm{C}$-shaped in $\mathrm{SCH}$. Figure is available in color online only.

the following parameters: $120 \mathrm{kV}$, dose modulation with variable milliamperes, and $2-\mathrm{mm}$ spatial resolution with coronal and sagittal reformatted images. All diagnoses were confirmed on surgery.

The imaging studies were analyzed by 2 board-certified neuroradiologists (P.R. and A.S.), both with more than 6 years of experience in diagnostic neuroimaging. The following features were assessed: 1) location of the deformity; 2) presence or absence of cord signal abnormality or syringomyelia; 3) visible arachnoid web; 4) presence of a dural defect; 5) nature of dorsal cord indentation (abrupt ["scalpel sign" as defined by Reardon et al. ${ }^{12}$ ] vs "C"shaped; Fig. 2); 6) focal ventral cord kink; 7) presence of the nuclear trail sign (endplate irregularity, sclerosis, and/ or disc-space calcification that could suggest a migratory path of a herniated disc); and 8) visualization of a complete plane of CSF ventral to the deformity.

\section{Results}

The age range for patients with DAWs was 18-67 years (4 males, 2 females), and 45-77 years ( 3 men, 2 women) for patients with SCH. DAWs were encountered between the T-3 and T-6 levels and SCHs were noted between the T-5 and T- 8 levels. Symptoms and clinical findings in both groups included back pain, lower-extremity weakness and paresthesia, erectile dysfunction, and fecal and urinary incontinence. One patient with SCH had Brown-Séquard syndrome. The frequencies of the above-described imaging findings are indicated in Table 1 . An arachnoid web was not definitively seen preoperatively in any patient with surgically proven DAWs. The dorsal cord indenta- 
TABLE 1. Imaging findings in DAWs and idiopathic SCH

\begin{tabular}{lcc}
\hline \multicolumn{1}{c}{ Imaging Finding } & DAW $(n=6)$ & $\mathrm{SCH}(\mathrm{n}=5)$ \\
\hline Location & T3-6 & T5-8 \\
\hline Cord T2 signal abnormality & 4 & $1^{*}$ \\
\hline Visible web & 2 & - \\
\hline Visible dural defect & 0 & 0 \\
\hline Scalpel sign & 6 & 0 \\
\hline C-shaped indentation & 0 & 5 \\
\hline Ventral cord kink & 0 & 3 \\
\hline Nuclear trail sign & 0 & 1 \\
\hline Ventral CSF plane preservation & 6 & 0 \\
\hline Ventral CSF plane interruption & 0 & 5 \\
\hline
\end{tabular}

* Cord signal was not assessed in 1 patient as only CT myelography was available.

tion demonstrated a scalpel-like appearance in all 6 patients with DAW and it was C-shaped in the 5 patients with $\mathrm{SCH}$. A focal ventral cord kink implying protrusion of the cord through a dural defect was observed in 3 of 5 patients with SCH and in none with DAW. In none of the patients was an actual dural defect identified. Signal intensity changes indicating myelomalacia, syringomyelia, or the pre-syrinx state were noted in 4 of 6 patients with DAW and in 1 patient with SCH (in 1 patient with $\mathrm{SCH}$, only myelographic images were available and the cord signal was not assessed). In 2 patients with the scalpel sign and syringomyelia, the syrinx was located below the level of the dorsal indentation. Given the hypothesis that a dural defect in some patients may be caused by a focal disc protrusion, ${ }^{11}$ the "nuclear trail" sign was also sought and was positive in 1 patient with SCH. A complete plane of CSF ventral to the subarachnoid space was visualized in all 6 patients with DAW. This was easier to determine on T2 SPACE and CT myelographic images. In all 5 patients with $\mathrm{SCH}$, this plane was found to be interrupted.

\section{Illustrative Cases \\ Case 1 (DAW)}

A 56-year-old man presented with a 3-month history of episodic urinary urgency, urinary intolerance, bowel incontinence, radicular pain, and gait difficulty. MRI of the thoracic spine showed expansion of the spinal cord from T-6 to T-10 with pre-syrinx conditions as well as a sudden change in cord caliber at the T-6 level with a scalpel-like dorsal indentation. T2-weighted SPACE and subsequent CT myelographic images did not show the arachnoid web directly. However, both demonstrated a complete plane of CSF ventral to the cord at the level of the deformity. Laminectomy revealed a large compressive DAW at T-7. Resection of the web led to an improvement in incontinence, radicular pain, and lower-extremity weakness (Fig. 3).

\section{Case 2 (SCH)}

A 77-year-old man presented with several years of lower-extremity weakness, numbness and paresthesia, and recent onset of urinary incontinence. On examination, decreased strength in the lower extremities and loss of sensa- tion in the right toes and perineum was noted. MRI, originally misinterpreted as a dorsal arachnoid cyst, revealed a focal cord deformity at T-5 with a C-shaped dorsal indentation and loss of the ventral CSF plane at the level of the deformity. He underwent a T5-6 laminectomy, release of the ventral herniation, dural repair, and T4-6 fusion, with recovery of sensation and motor function (Fig. 4).

\section{Discussion}

Our findings revealed that DAW and SCH can be reliably distinguished on imaging by scrutinizing the nature of the dorsal indentation and the integrity of the ventral subarachnoid space at the level of the cord deformity. The septum posticum of Schwalbe traverses the posterior spinal subarachnoid space in the sagittal plane and is especially well developed in the thoracic spinal canal where it presumably serves to stabilize the cord. It is sometimes seen as a filling defect on supine myelograms. ${ }^{4}$ The septum is believed to progressively degenerate with arachnoid cysts, with webs and adhesions representing its remnants. These may compress the cord and impede CSF flow to varying degrees. ${ }^{8}$ Most cases of $\mathrm{SCH}$ are idiopathic. They may arise from remote trauma leading to disruption of the dura or in some instances from a dural rent caused by a disc protrusion ${ }^{11}$ with subsequent tamponade by the cord. ${ }^{2}$ Rare instances of cord herniation into congenital dural defects and of herniated cord tissue trapped between 2 layers of congenital dural duplication have also been reported..$^{14,17}$ An as-yet undetermined inflammatory process leading to adhesion of the cord to the ventral dura and subsequent development of a dural defect arising from pressure erosion by the pulsating, adherent cord has also been proposed as a mechanism for development of SCH..$^{10}$ The clinical presentation of both entities is similar with symptoms including back, neck, or shoulder pain; upper- and lower-extremity paresthesias and weakness; and BrownSéquard syndrome. ${ }^{11,12}$ Symptoms may be due to a combination of direct compression of the cord and development of syringomyelia, presumably resulting from impairment of CSF flow.

Surgical treatment of DAW involves laminectomy with intradural lysis of adhesions and resection of arachnoid bands. Careful attention must be given to verify that there is satisfactory flow of CSF from the cranial and caudal ends of the dural opening. If there is residual arachnoid membrane present, at the caudal or cephalad aspects of the dural opening, we advocate suturing this arachnoid layer to the residual dura during dural closure to prevent CSF from accumulating into a potential space between the arachnoid and the dura. The primary aims of $\mathrm{SCH}$ repair include reduction of the herniation, restoration of the spinal cord to its normal position, and prevention of recurrence of herniation. ${ }^{16}$ There appear to be 3 approaches toward these ends: some advocate primary closure of the dural defect, some close the dural defect with fat or fascia, and others prefer to enlarge the dural defect. All of these require inspection of the ventral cord surface by dividing the dentate ligaments. Complications from such procedures may include anterior epidural CSF collection and superficial siderosis. ${ }^{5}$ 

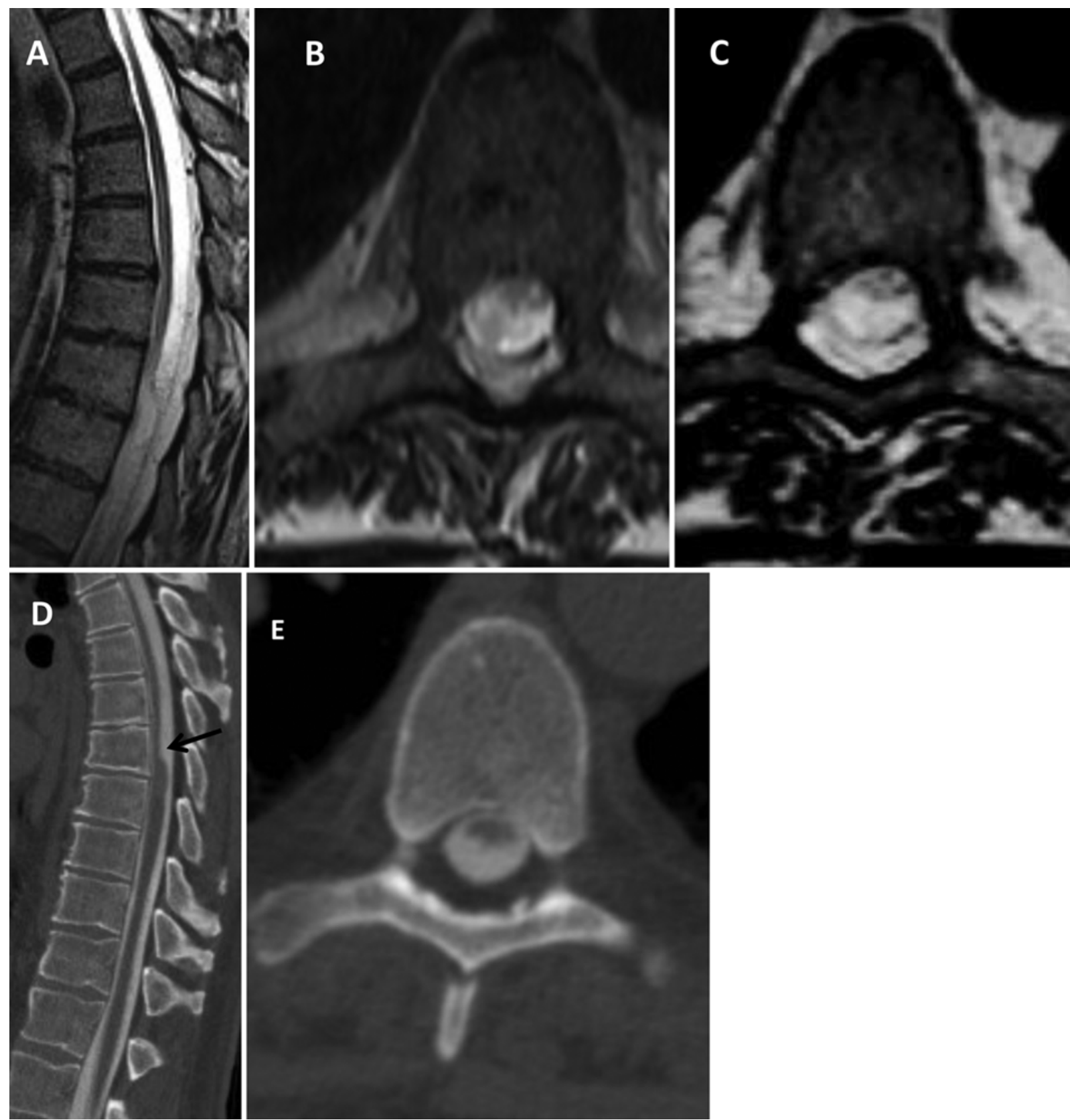

FIG. 3. Case 1. DAW. Sagittal T2 SPACE (A), axial T2-weighted MR image (B), axial reformat of the sagittal T2 SPACE (C), and sagittal (D) and axial (E) myelographic images from the same 56-year-old man with midthoracic DAW that showed a scalpel sign with cord expansion below the level of deformity (best seen on $A$ and $D$ ). The preserved ventral subarachnoid space is not well seen on the axial T2-weighted MR image but is readily appreciated on the axial reformat (arrow, D) and the axial myelographic image $(\mathrm{E})$, excluding $\mathrm{SCH}$. Note that the web itself is not demonstrated.

Direct visualization of the DAW remains difficult using conventional imaging techniques. A web was not confidently identified in any of the 14 cases reported by Reardon et al. ${ }^{12}$ and was identified in only 2 of 7 cases by Hakky et al. ${ }^{8}$ Improved visualization of these webs may be achieved by the addition of "myelographic" high-resolution T2-weighted MRI sequences ${ }^{6}$ such as constructive interference in steady state (CISS) or SPACE, or their equivalents (Fig. 3). Despite the use of the SPACE sequence in 4 patients in our series, a web was not definitively identified in any of these patients. The dural defect and focally her- niated ventral cord parenchyma may also be difficult to identify. A dural defect was not identifiable in any of our patients and a ventral cord deformity was present in only 3 of 5 patients with $\mathrm{SCH}$.

Preoperative imaging differentiation between these entities is therefore best achieved by identifying indirect signs. The 2 most reliable signs to differentiate DAW from SCH were 1) the shape of the dorsal indentation on sagittal MRI or CT myelography, and 2) the presence or absence of a plane of CSF ventral to the cord. The scalpel sign described by Reardon et al. refers to a characteristic notch 

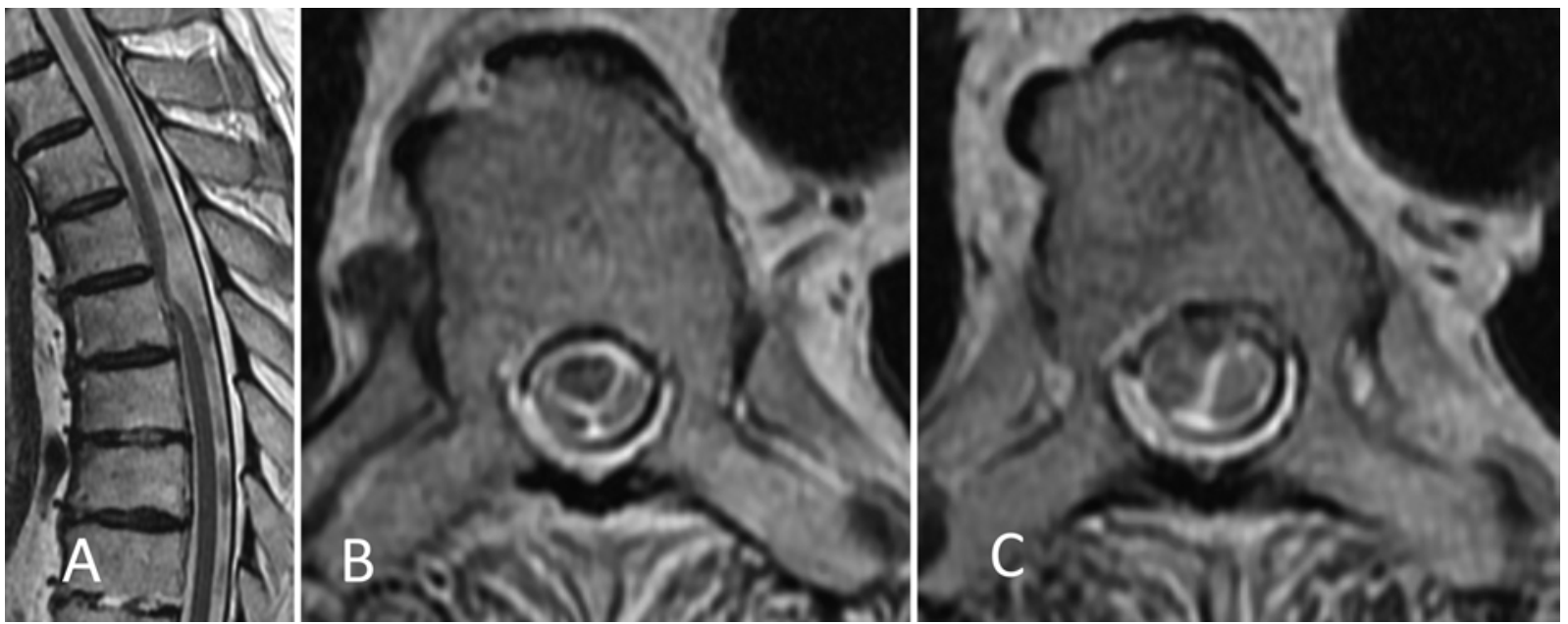

FIG. 4. Case 2. SCH. Sagittal (A) and axial T2-weighted MRI above (B) and at the level of cord deformity (C) show a focal Cshaped indentation of the posterior midthoracic cord. Note the lack of visible CSF signal and interruption of the ventral subarachnoid space anterior to the ventral cord (C).

such as focal dorsal indentation of the upper thoracic spinal cord on sagittal MRI and CT myelography, resulting in the cord resembling a scalpel with its blade pointing posteriorly. ${ }^{12}$ This arises from displacement and compression of the cord by the arachnoid band in the dorsal subarachnoid space and was present in all cases of DAW in our series. In all patients with $\mathrm{SCH}$, the indentation was C-shaped, due to a short segment of the cord being pulled ventrally, distal to which it returns to its normal position (Figs. 1 and 2). Close scrutiny of the ventral subarachnoid space is also warranted. In our series, this space was better identified on T2 SPACE images and was preserved in all cases of DAW and interrupted in all cases of SCH (Fig. 3 ). This is an intuitive but crucial finding given that a ventral cord herniation will, by definition, lead to interruption of the CSF plane. Although it is conceivable that a DAW may produce such severe mass effect to interrupt this CSF plane, we did not observe this in any of our patients. It is important to note that evaluation of the ventral CSF space also may be limited by the slice thickness and resolution of standard MRI sequences. We suggest that high-resolution MRI be first performed to delineate the ventral subarachnoid space and a CT myelogram be used as the next step in the eventuality of the MRI being inconclusive. Our case series illustrates the value of high-resolution imaging via either T2 SPACE images or CT myelography in those instances in which conventional MRI is equivocal.

\section{Study Limitations}

The retrospective nature of our study and the small sample size are important limitations. The full gamut of standard and high-resolution MRI sequences was not available for all our patients, which limited a direct comparison of the accuracies of MRI and CT myelography. Also, no CSF flow-sensitive sequences were obtained in any of our patients. These may have some value in identifying the site of CSF flow interruption. For example, Chang et al. reported the use of cardiac-gated, phase-contrast, cine-mode MRI to precisely localize webs in 2 patients. ${ }^{3}$ Brugières et al. ${ }^{1}$ employed phase-contrast MRI to demonstrate the absence of flow ventrally with preserved flow dorsally in 2 patients with $\mathrm{SCH}$. We also did not include arachnoid cysts in our analysis. Arachnoid cysts are recognized by their marginated walls, smooth wide scalloping of the dorsal cord surface, and their tendency to present as slowly filling defects compared with the remainder of the subarachnoid space on myelography. ${ }^{12}$

\section{Conclusions}

In summary, DAW and SCH both present as focal deformities of the thoracic spinal cord on imaging, but their differentiation has important surgical implications. In our small series, imaging reveals that the scalpel-shaped dorsal indentation and preservation of the ventral CSF plane are strongly suggestive of DAW while the $\mathrm{C}$-shaped deformity and interruption of the ventral CSF appear to be typical of SCH. Although conventional MRI may be adequate in the characterization of these findings, high-resolution T2-weighted sequences and CT myelography are useful problem-solving tools.

\section{Acknowledgments}

We would like to thank Brigitte Pocta, MLA, for her assistance with preparation of this manuscript.

\section{References}

1. Brugières P, Malapert D, Adle-Biassette H, Fuerxer F, Djindjian M, Gaston A: Idiopathic spinal cord herniation: value of MR phase-contrast imaging. AJNR Am J Neuroradiol 20:935-939, 1999

2. Brus-Ramer M, Dillon WP: Idiopathic thoracic spinal cord herniation: retrospective analysis supporting a mechanism of diskogenic dural injury and subsequent tamponade. AJNR Am J Neuroradiol 33:52-56, 2012

3. Chang HS, Nagai A, Oya S, Matsui T: Dorsal spinal arachnoid web diagnosed with the quantitative measurement of cerebrospinal fluid flow on magnetic resonance imaging. J Neurosurg Spine 20:227-233, 2014 
4. Di Chiro G, Timins EL: Supine myelography and the septum posticum. Radiology 111:319-327, 1974

5. Gaudino S, Colantonio R, Schiarelli C, Martucci M, Calandrelli R, Botto A, et al: Postoperative MR imaging of spontaneous transdural spinal cord herniation: expected findings and complications. AJNR Am J Neuroradiol 37:558-564, 2016

6. Grewal SS, Pirris SM, Vibhute PG, Gupta V: Identification of arachnoid web with a relatively novel magnetic resonance imaging technique. Spine J 15:554-555, 2015

7. Haber MD, Nguyen DD, Li S: Differentiation of idiopathic spinal cord herniation from CSF-isointense intraspinal extramedullary lesions displacing the cord. Radiographics 34:313-329, 2014

8. Hakky MM, Justaniah AI, David C, French RJ, Martin D, Kwok N, et al: The neuroimaging spectrum of septum posticum derangement and associated thoracic myelopathy. $\mathbf{J}$ Neuroimaging 25:818-823, 2015

9. Isu T, Iizuka T, Iwasaki Y, Nagashima M, Akino M, Abe H: Spinal cord herniation associated with an intradural spinal arachnoid cyst diagnosed by magnetic resonance imaging. Neurosurgery 29:137-139, 1991

10. Najjar MW, Baeesa SS, Lingawi SS: Idiopathic spinal cord herniation: a new theory of pathogenesis. Surg Neurol 62:161-171, 2004

11. Parmar H, Park P, Brahma B, Gandhi D: Imaging of idiopathic spinal cord herniation. Radiographics 28:511-518, 2008

12. Reardon MA, Raghavan P, Carpenter-Bailey K, Mukherjee S, Smith JS, Matsumoto JA, et al: Dorsal thoracic arachnoid web and the "scalpel sign": a distinct clinical-radiologic entity. AJNR Am J Neuroradiol 34:1104-1110, 2013

13. Slavotinek JP, Sage MR, Brophy BP: An unusual spinal intradural arachnoid cyst. Neuroradiology 38:152-154, 1996

14. Sugimoto T, Kasai Y, Takegami K, Morimoto R, Maeda M, Uchida A: A case of idiopathic spinal cord herniation with duplicated dura mater. J Spinal Disord Tech 18:106-111, 2005

15. Tekkök IH: Spontaneous spinal cord herniation: case report and review of the literature. Neurosurgery 46:485-492, 2000

16. Watanabe M, Chiba K, Matsumoto M, Maruiwa H, Fujimura Y, Toyama Y: Surgical management of idiopathic spinal cord herniation: a review of nine cases treated by the enlargement of the dural defect. J Neurosurg 95 (2 Suppl):169-172, 2001

17. Yamamoto N, Katoh S, Higashino K, Sairyo K: Idiopathic spinal cord herniation with duplicated dura mater and dorsal subarachnoid septum. Report of a case and review of the literature. Int J Spine Surg 8:29, 2014

\section{Disclosures}

Dr. Sansur has served as a consultant to Medtronic, Globus, Stryker, and DePuy Synthes.

\section{Author Contributions}

Conception and design: Raghavan. Acquisition of data: Raghavan, Schultz, Steven, Gandhi. Analysis and interpretation of data: Raghavan, Schultz, Steven, Fischbein. Drafting the article: Raghavan, Schultz, Steven, Wessell, Fischbein, Sansur, Ibrahimi. Critically revising the article: all authors. Reviewed submitted version of manuscript: all authors. Approved the final version of the manuscript on behalf of all authors: Raghavan. Study supervision: Raghavan.

\section{Correspondence}

Prashant Raghavan, Department of Diagnostic Radiology and Nuclear Medicine, University of Maryland Medical Center, 22 South Greene St., Baltimore, MD 21201. email: praghavan@ umm.edu. 